\title{
Квантовый выход кремниевого лавинного фотодиода в диапазонах длин волн 114-170 и 210-1100 nm
}

\author{
(ㄷ П.Н. Аруев, В.П. Белик, А.А. Блохин, В.В. Забродский, А.В. Николаев, В.И. Сахаров, И.Т. Серенков, \\ В.В. Филимонов, Е.В. Шерстнев
}

Физико-технический институт им. А.Ф. Иофффе РАН, Санкт-Петербург, Россия

E-mail: sildet@mail.ioffe.ru

Поступило в Редакцию 17 сентября 2021 г.

В окончательной редакции 19 ноября 2021 г.

Принято к публикации 29 ноября 2021 г.

\begin{abstract}
Разработан кремниевый лавинный фотодиод для регистрации ближнего инфракрасного, видимого, ультрафиолетового и вакуумного ультрафиолетового спектров. Проведено исследование внешнего квантового выхода кремниевого лавинного фотодиода в диапазоне длин волн $114-170$ и 210-1100 nm. Показано, что лавинный фотодиод обладает внешним квантовым выходом от 29 до 9300 electrons/photon на длине волны $160 \mathrm{~nm}$ при напряжении обратного смещения 190-303 V соответственно.
\end{abstract}

Ключевые слова: лавинный фотодиод, вакуумный ультрафиолет, видимый диапазон, ближний инфракрасный диапазон, кремний.

DOI: 10.21883/PJTF.2022.05.52146.19026

Ранее нами был представлен $n^{++}-p-\pi-p^{++}$-кремниевый лавинный фотодиод (ASPD) с внешним квантовым выходом $\eta_{\text {ASPD }}=24-150$ electrons/photon в спектральном диапазоне $120-170 \mathrm{~nm}$ [1]. Для увеличения $\eta_{\text {ASPD }}$ в вакуумном ультрафиолете нами был разработан и исследован фотодиод ASPD c оптимизированной структурой.

Как показано в [1], применение традиционных антиотражающих покрытий на основе диэлектриков $\left(\mathrm{SiO}_{2}\right.$, $\left.\mathrm{MgF}_{2}, \mathrm{LiF}, \mathrm{Si}_{3} \mathrm{~N}_{4}\right)$ является неприемлемым для длин волн $\lambda<130 \mathrm{~nm}$ ввиду резкого уменьшения глубины поглощения квантов до значения $\sim 10 \mathrm{~nm}$. Такая глубина поглощения квантов вакуумного ультрафиолета (ВУФ) делает любые диэлектрические антиотражающие покрытия непрозрачными для $20<\lambda<130 \mathrm{~nm}$ [2]. В качестве альтернативы антиотражающим покрытиям в конструкции нового ASPD был применен известный метод текстурирования поверхности активной области [3]. Это было сделано для уменьшения потерь, связанных с отражением ВУФ. Дополнительно была уменьшена температура диффузии бора при формировании изотипного $p^{++}$ слоя на текстурированной поверхности. Это было сделано для увеличения эффективности регистрации ВУФ за счет уменьшения глубины залегания изотипного $p^{++}-\pi$ перехода. Кроме того, для снижения темнового тока была уменьшена толщина активной области фотодиода до $150 \mu \mathrm{m}$ методом реактивного ионного травления. В остальном конструкция ASPD, оптимизированного для регистрации ВУФ, была аналогична конструкции ASPD из работы [1].
Оптимизированный ASPD в режимах работы „front

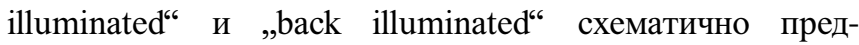
ставлен на рис. 1. В настоящей работе в режиме „front illuminated“ ASPD облучался потоком квантов в спектральном диапазоне $\lambda=600-1100 \mathrm{~nm}$ со стороны $n^{++}$-слоя, покрытого нитридом кремния (рис. $1, a$ ). При облучении в режиме „back illuminated“ ASPD облучался потоком квантов в спектральных диапазонах $\lambda=114-170$ и 210-1100 nm со стороны $p^{++}$-слоя (рис. $1, b)$. Смена режимов облучения ASPD достигалась переворотом подкристальной платы и фиксацией ее методом пайки к выводам корпуса ТО-5 (рис. 1,c).

Спектральные зависимости $\eta_{\mathrm{ASPD}}$ для режимов работы „front illuminated“" и „back illuminated“ исследовались на основе метрологической базы из работы [1] при напряжении обратного смещения $280 \mathrm{~V}$ и температуре $22^{\circ} \mathrm{C}$. В случае диапазона $\lambda=114-170 \mathrm{~nm}$ размер выходной щели вакуумного монохроматора в настоящей работе был уменьшен с $0.3 \times 0.3$ до $0.15 \times 0.15 \mathrm{~mm}$. Это было сделано для гарантированного попадания всего пучка ВУФ в активную область ASPD диаметром $1 \mathrm{~mm}$. Данное условие является необходимым при измерении спектральных характеристик методом сравнения. Значение $\eta_{\mathrm{ASPD}}$ определялось выражением

$$
\eta_{\mathrm{ASPD}}=\eta_{\mathrm{SPD}}\left(I_{\mathrm{ASPD}} / I_{\mathrm{SPD}}\right)
$$

где $\eta_{\mathrm{SPD}}$ - внешний квантовый выход калиброванного фотодиода, $I_{\mathrm{ASPD}}-$ фототок ASPD, $I_{\mathrm{SPD}}-$ фототок калиброванного фотодиода. В качестве калиброванного использовался фотодиод SPD без внутреннего усиления [4]. Результаты представлены на рис. 2. 

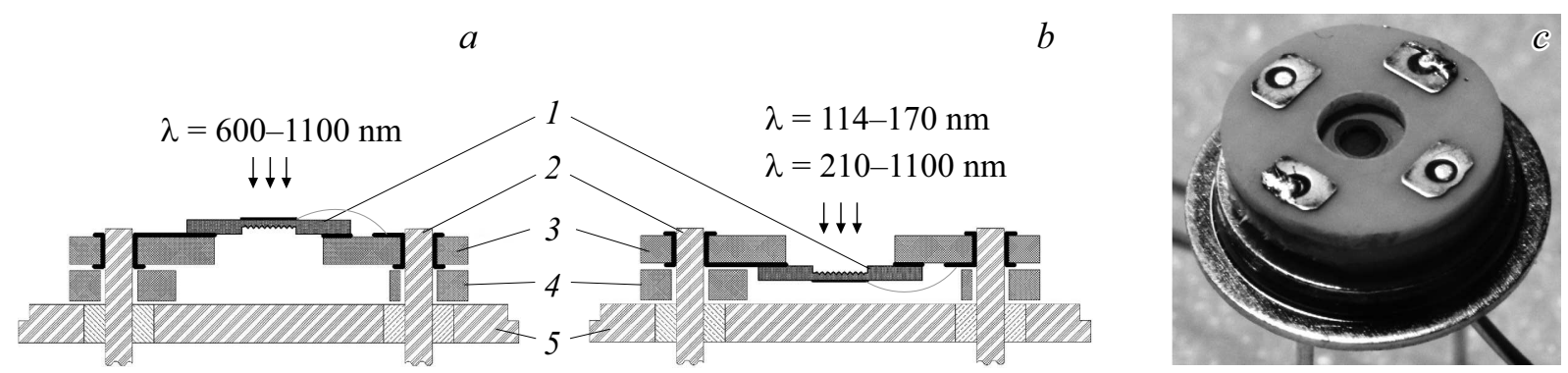

Рис. 1. ASPD в корпусе TO-5. $a-$ режим „front illuminated“, $b-$ режим „back illuminated“ $(1-$ кристалл ASPD, 2 электрически изолированные выводы корпуса, 3 - подкристальная диэлектрическая плата с металлизацией, 4 - диэлектрическая плата, 5 - основание металлостеклянного корпуса TO-5), $c$ - фотография ASPD в корпусе TO-5 в режиме „back illuminated“.

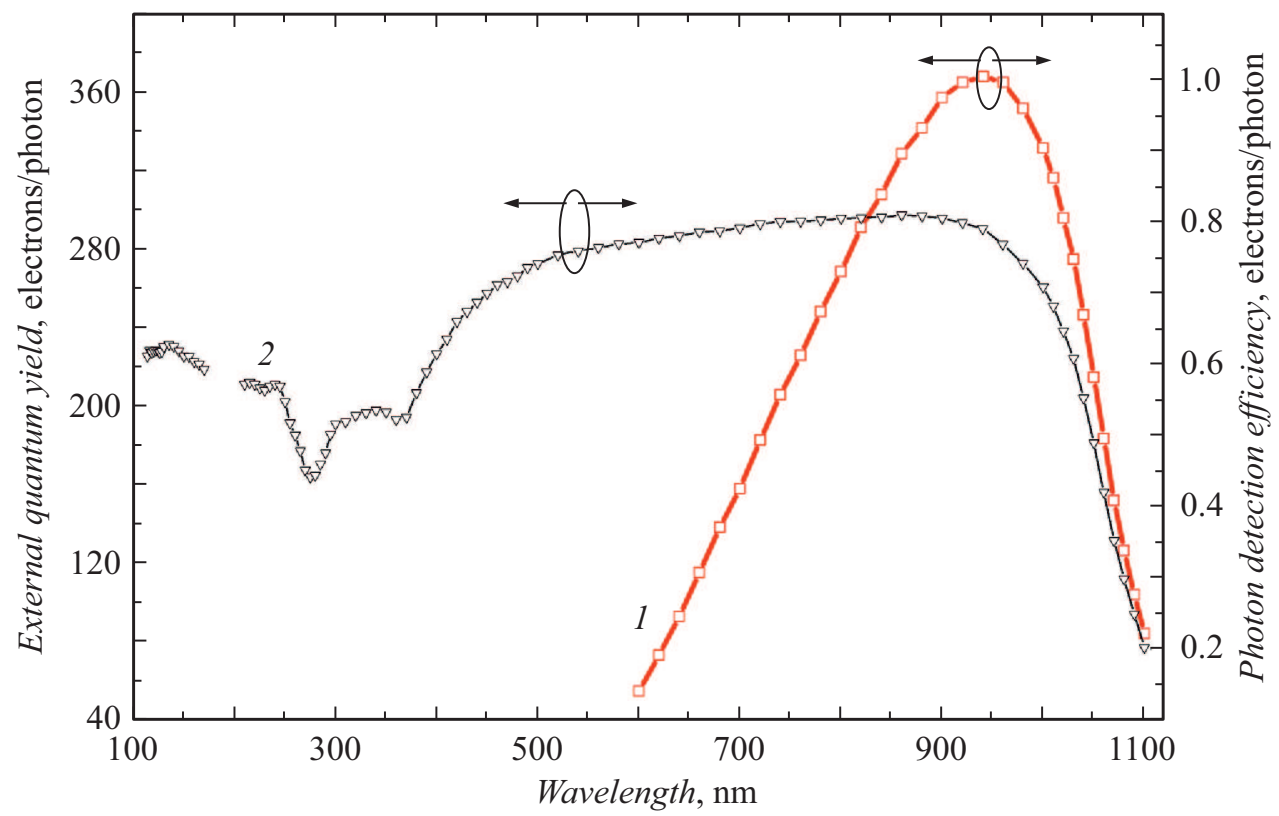

Pис. 2. Спектральные зависимости внешнего квантового выхода ASPD и эффективности регистрации фотонов ASPD в режимах „front illuminated“ (1) и „back illuminated“ (2).

Методологическое отличие настоящей работы от [1] заключалось в определении коэффициента умножения ASPD $\left(M_{\mathrm{ASPD}}\right)$. Чтобы определить $M_{\mathrm{ASPD}}$, была использована спектральная зависимость $\eta_{\mathrm{ASPD}}$ в режиме „front illuminated“ (рис. 2). В этом режиме облучение ASPD происходило со стороны $n^{++}$-слоя, покрытого нитридом кремния толщиной $0.12 \mu \mathrm{m}$. Поскольку толщина нитрида кремния на поверхности $n^{++}$-слоя известна из технологии изготовления с точностью не меньше $2 \%$, из преобразований Френеля [5] следует, что потери на отражение для $\lambda=940 \mathrm{~nm}$ будут меньше $1 \%$, а значит, этими потерями можно пренебречь. Это допущение позволяет в дальнейшем исходить из того, что 100\% излучения будет поглощаться в толщине активной области ASPD (для $\lambda=940 \mathrm{~nm}$ в случае режима „front illuminated“). Из технологии изготовления ASPD известно, что толщина $n^{++}$-слоя $\sim 0.5 \mu \mathrm{m}$, суммарная толщина $p$ - и $\pi$-областей $\sim 150 \mu \mathrm{m}$, толщина $p^{++}$-слоя $\sim 0.03 \mu \mathrm{m}$. Поскольку глубина поглощения излучения с $\lambda=940 \mathrm{~nm}$ в кремнии $\sim 40 \mu \mathrm{m}[2]$, не менее 99\% этого излучения поглотится в $p$ - и $\pi$-областях. Это обусловлено тем, что потери данного излучения в $n^{++}$-слое будут на уровне десятых долей процента, а до $p^{++}$-слоя излучение с этой длиной волны дойдет в еще меньшем количестве. Выше было показано, что в случае $\lambda=940 \mathrm{~nm}$ потери на отражение близки к нулю, тогда с учетом распределения излучения с этой длиной волны в структуре ASPD можно утверждать, что в случае режима „front illuminated“ при $\lambda=940 \mathrm{~nm}$ величина $M_{\mathrm{ASPD}}$ совпадает с $\eta_{\mathrm{ASPD}}$ на $99 \%$ :

$$
M_{\mathrm{ASPD}}=\eta_{\mathrm{ASPD}}
$$

где $\eta_{\mathrm{ASPD}}-$ внешний квантовый выход ASPD, вычисленный по формуле (1). В настоящей работе значение $M_{\mathrm{ASPD}}$ было равно 366 (рис. 2) для режима „front illuminated“ при $\lambda=940 \mathrm{~nm}$. Для расчетов в режиме „back illuminated“ использовалось значение $M_{\mathrm{ASPD}}=366$, определенное в режиме „front illuminated“ при $\lambda=940 \mathrm{~nm}$, так как $M_{\mathrm{ASPD}}$ считаем характеристикой 


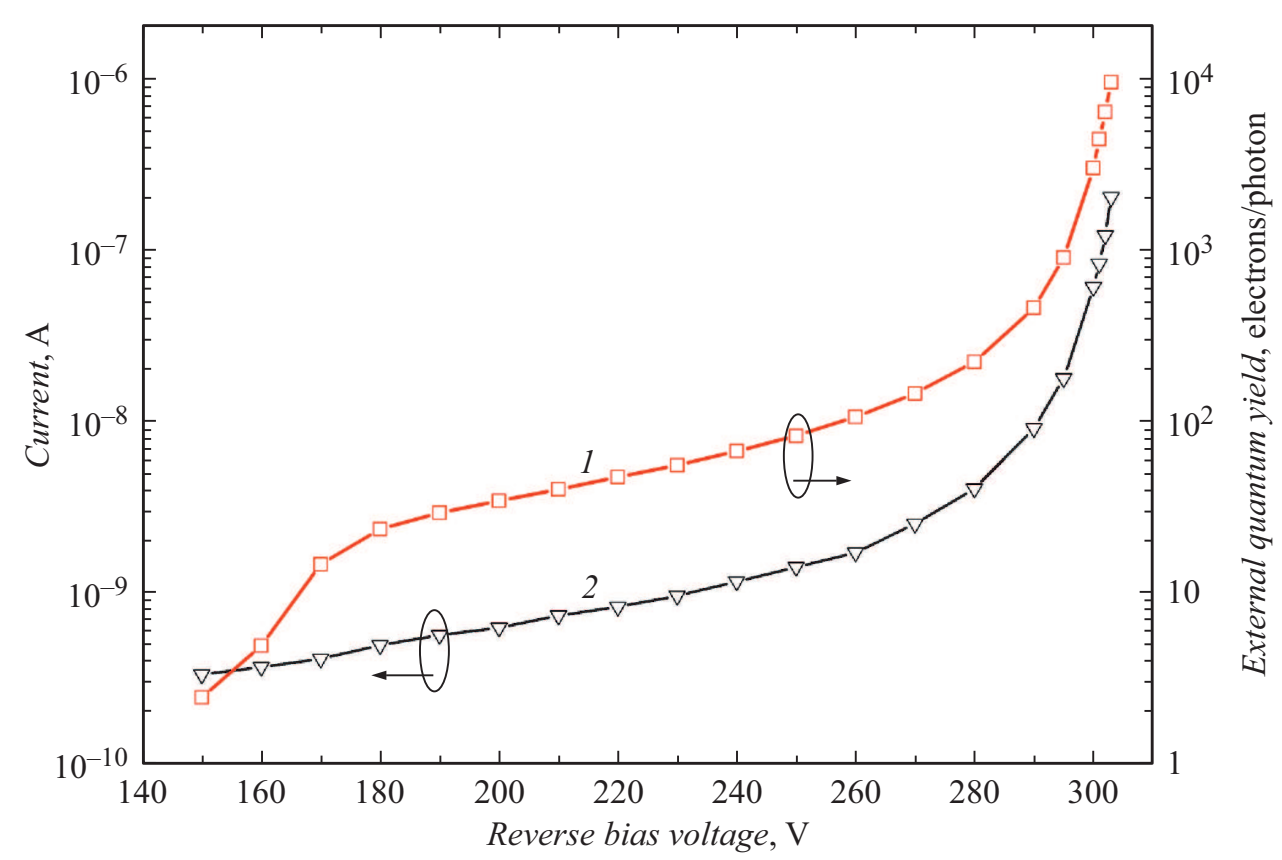

Рис. 3. Зависимости характеристик ASPD в режиме „back illuminated“ от напряжения обратного смещения. 1 - внешний квантовый выход $(\lambda=160 \mathrm{~nm}), 2$ - темновой ток.

$n^{++}-p$-перехода, а значит, функцией температуры и напряжения обратного смещения на переходе, которые были одинаковы для режимов „front illuminated“ и „,back illuminated“. На основе известных значений $\eta_{\text {ASPD }}$ и $M_{\mathrm{ASPD}}$ были вычислены спектральные зависимости эффективности регистрации фотонов ASPD $\left(\varepsilon_{\mathrm{ASPD}}\right)$ для режимов облучения „front illuminated“" и „,back illuminated“. Величина $\varepsilon_{\mathrm{ASPD}}$ определялась выражением

$$
\varepsilon_{\mathrm{ASPD}}(\lambda)=\eta_{\mathrm{ASPD}}(\lambda) / M_{\mathrm{ASPD}}
$$

На рис. 2 также приведены значения $\varepsilon_{\mathrm{ASPD}}$ в режимах „front illuminated“ и „,back illuminated“.

Для оценки доступного диапазона значений $\eta_{\mathrm{ASPD}}$ в режиме „back illuminated“ в области вакуумного ультрафиолета была выбрана длина волны $160 \mathrm{~nm}$. На этой длине волны плотность излучения газоразрядной лампы максимальна, что удобно с метрологической точки зрения. Величина $\eta_{\mathrm{ASPD}}$ определялась по формуле (1). На рис. 3 представлены зависимости $\eta_{\mathrm{ASPD}}$ и темнового тока ASPD от напряжения обратного смещения.

Из экспериментальных данных видно, что изотипный $p^{++}-\pi$-переход на текстурированной поверхности активной области кремниевого лавинного фотодиода позволяет обеспечить внешний квантовый выход от 29 до 9300 electrons/photon на длине волны $160 \mathrm{~nm}$. Оптимизированный ASPD в режиме „,back illuminated“ демонстрирует эффективность регистрации фонов 0.59-0.63 electrons/photon в диапазоне длин волн $114-170 \mathrm{~nm}$ и 0.44-0.81 electrons/photon в диапазоне длин волн 210-1050 nm. Эффективность регистрации фотонов исследованного ASPD в диапазонах длин волн 114-130 и
275-1050 nm превосходит эффективность фотодиода из работы [6].

Таким образом, в работе исследован ASPD, оптимизированный для регистрации вакуумного ультрафиолета. Показано, что за счет уменьшения потерь на отражение методом текстурирования активной области и уменьшения глубины залегания изотипного $p^{++}-\pi$-перехода внешний квантовый выход и эффективность регистрации ВУФ фотонов были увеличены более чем в 4.5 раза относительно аналогичных характеристик ASPD из нашей предыдущей работы [1].

\section{Благодарности}

Авторы благодарны коллегам из ФТИ им. А.Ф. Иоффе Н.В. Забродской, М.С. Лазеевой, М.В. Дроздовой, В.И. Маршаловой за помощь в изготовлении фотодиодов.

\section{Конфликт интересов}

Авторы заявляют, что у них нет конфликта интересов.

\section{Список литературы}

[1] П.Н. Аруев, В.П. Белик, В.В. Забродский, Е.М. Круглов, А.В. Николаев, В.И. Сахаров, И.Т. Серенков, В.В. Филимонов, Е.В. Шерстнев, ЖТФ, 90 (8), 1386 (2020). DOI: 10.21883/JTF.2020.08.49552.44-20 [P.N. Aruev, V.P. Belik, V.V. Zabrodskii, E.M. Kruglov, A.V. Nikolaev, V.I. Sakharov, I.T. Serenkov, V.V. Filimonov, E.V. Sherstnev, Tech. Phys., 65 (8), 1333 (2020).

DOI: $10.1134 / \mathrm{S} 1063784220080022]$. 
[2] Handbook of optical constants of solids, ed by E.D. Palik (Academic Press, USA, 1998).

[3] H. Schröder, E. Obermeier, A. Steckenborn, J. Micromech. Microeng., 9 (2), 139 (1999).

DOI: $10.1088 / 0960-1317 / 9 / 2 / 309$

[4] П.Н. Аруев, С.В. Бобашев, А.М. Красильщиков, А.В. Николаев, Д.Ю. Петров, Е.В. Шерстнев, ПТЭ, № 1, 98 (2021). DOI: 10.31857/S003281622006018X [P.N. Aruev, S.V. Bobashev, A.M. Krassilchtchikov, A.V. Nikolaev, D.Yu. Petrov, E.V. Sherstnev, Instrum. Exp. Tech., 64 (1), 93 (2021). DOI: 10.1134/S0020441220060147].

[5] M. Born, E. Wolf, Principles of optics, 7th ed. (Cambridge University Press, 1999). DOI: 10.1017/CBO9781139644181

[6] R. Chandrasekharan, M. Messina, A. Rubbia, Nucl. Instrum. Meth. Phys. Res. A, 567 (1), 45 (2006). 